\title{
Culture et consommation dans une communauté guarani-ñandeva du Chaco
}

Jean-Pierre Estival

\section{(2) OpenEdition \\ 12 Journals}

Édition électronique

URL : https://journals.openedition.org/tc/1276

DOI : $10.4000 /$ tc. 1276

ISSN : 1952-420X

Éditeur

Éditions de l'EHESS

\section{Édition imprimée}

Date de publication : 1 mars 2002

ISSN : 0248-6016

\section{Référence électronique}

Jean-Pierre Estival, «Culture et consommation dans une communauté guarani-ñandeva du Chaco », Techniques \& Culture [En ligne], 38 | 2002, mis en ligne le 12 juin 2006, consulté le 29 septembre 2022. URL : http://journals.openedition.org/tc/1276 ; DOI : https://doi.org/10.4000/tc.1276

Ce document a été généré automatiquement le 29 septembre 2022.

Tous droits réservés 


\title{
Culture et consommation dans une communauté guarani-ñandeva du Chaco
}

\author{
Jean-Pierre Estival
}

1 Lorsque l'on aborde la vie quotidienne d'un village amérindien -le barrio San Lázaro de Santa Teresita- établi depuis plus de cinquante ans dans une mission catholique, de surcroît près d'une ville et au bord de la seule route asphaltée de la région, la première question qui vient à l'esprit est «Qu'est-ce qui différencie ce village d'une communauté du Chaco peuplée de paysans pauvres néo-américains (i.e. non indiens) ?». Bien sûr, on va vite constater qu'ils vivent dans une précarité encore plus grande... Néanmoins, arrivé sur place pour étudier les patrimoines musicaux et chorégraphiques amérindiens, et avec mon regard ethnologique sans doute un peu classique, les manques de différenciation culturelle par rapport à la société englobante me surprirent lors de mes premiers séjours :

2 - la langue originale -connue de tous mais cachée scrupuleusement aux Paraguayens non amérindiens- est remplacée dans la vie quotidienne par le guarani jopara, langue vernaculaire du Paraguay, tant chez les Néo-américains que chez les Amérindiens de souche guarani (on parle alors de guarani indigène pour les langues différenciées de chaque culture guarani, qui peuvent être fort différentes du guarani jopara, comme c'est le cas chez les Guarani-Ñandeva1);

- la culture matérielle ancienne a presque complètement disparu, la nourriture traditionnelle aussi, remplacée par le riz, les galettes de farine de blé frites et les pâtes;

- l'agriculture, l'élevage, la chasse et la cueillette ne suffisent plus -loin s'en faut-à assurer la subsistance;

- il n'y a plus de chamanes importants;

- les termes de parenté sont espagnols (prima, tio) ou guarani jopara;

- et apparemment, on ne pratique plus ni rituels ni musiques.

3 Ainsi, comment qualifier les Guarani-Ñandeva d'aujourd'hui, autrement qu'en termes de catégorisation néo-américaine externe -ce sont des indigènes (conformément à 
l'histoire et à la loi 904/81)² - ou de revendication politique interne? Les premières réponses à ces constatations préliminaires peuvent être approchées par l'analyse de la structure du village et de la circulation des biens, et en particulier de ceux qui touchent directement à l'alimentation.

Les peuples autochtones du Chaco sont classés traditionnellement parmi les chasseurscueilleurs, mais l'on constatera que d'une part, cela n'a jamais vraiment été le cas des Ñandeva, et que d'autre part ces deux activités sont aujourd'hui tout à fait marginales dans leurs pratiques de subsistance.

Dans le sillage des études d'Alvarsson (1999), Von Bremen (1991), Picon (1998) et Dousset (1999) -ce dernier pour une tout autre aire culturelle, mais avec une problématique comparable-, nous tenterons de montrer comment, à partir du fonds traditionnel d'avant le contact (années 1930), une communauté guarani-ñandeva continue de cultiver une forte idéologie égalitaire, avec des modes de production et des biens qui ont radicalement changé, comme on va le voir maintenant.

Les Guarani-Ñandeva forment l'unique composante purement chaquéenne de la grande famille linguistique tupi-guarani. La carte de la figure 1 montre approximativement leur situation géographique traditionnelle au premier tiers du vingtième siècle, celle des Amérindiens présents dans leur mémoire (leurs voisins de l'époque, avec qui se sont effectuées les interactions que nous évoquerons), ainsi que l'emplacement actuel de Santa Teresita.

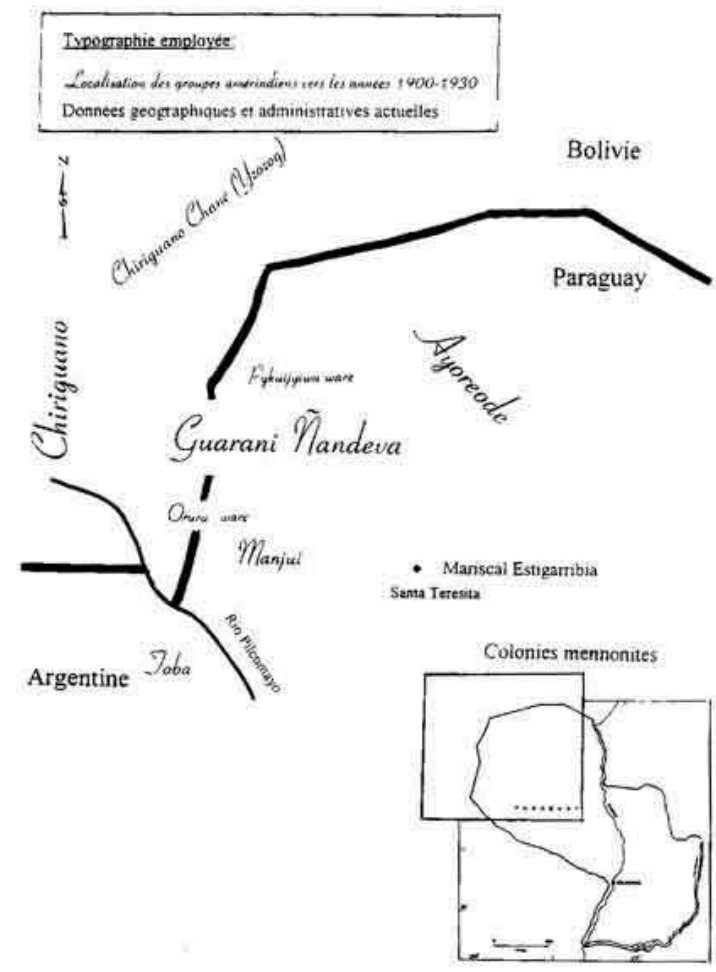

7 On a vu que leur langue originale, non encore décrite, est gardée plus ou moins secrète pour les étrangers, et particulièrement pour les Paraguayens. C'est incontestablement une langue tupi-guarani (vocabulaire, syntaxe), plus proche du chiriguano que du guarani paraguayen de la région orientale. Un survol de l'étude de référence de Dietrich (1992) montre néanmoins de notables différences avec le chiriguano. Ces différences sont telles qu'il n'est pas exclu de penser que les Ñandeva forment un 
rameau séparé depuis longtemps des Chiriguano. Une intercompréhension "de bas » reste néanmoins possible, ce qui n'est pas le cas avec le guarani jopara paraguayen.

La société ñandeva était semi-nomade, les villages étant établis pour quelques années à proximité de terres cultivables et de points d'eau douce. Les guerres avec les Ayoréo (Tsirarakwa) et les Chiriguano, principalement, puis celles avec les militaires boliviens à partir de la fin du XIXe siècle, pouvaient également provoquer des déplacements de population; depuis une cinquantaine d'années, les villages sont fixes, même si les individus se déplacent beaucoup, en particulier pour chercher du travail.

9 À l'instar des autres peuples du Chaco, ils sont considérés comme des (ex)chasseurscueilleurs ou collecteurs-chasseurs ${ }^{3}$. En fait, on verra que le témoignage des anciens ayant connu la vie d'avant le contact permanent avec les Néo-américains - soit avant la guerre du Chaco de 1932-35- pousserait plutôt à envisager cette culture comme celle d'agriculteurs-éleveurs-collecteurs-chasseurs, si tant est que ce type de classification soit encore pertinent. Rappelons que l'élevage des animaux d'origine européenne (moutons, chèvres, chevaux et bovins), pratiqué dans le Chaco par de nombreux groupes amérindiens, remonte sans doute à la fin du XVIIe siècle (Métraux 1946), alors même que ces sociétés n'avaient pas ou avaient peu de contacts directs, si ce n'est belliqueux, avec les Espagnols.

10 Diverses hypothèses (Del Campana 1902, Nordenskiöld 1910, Schmidt 1937) ont été formulées sur leur origine: l'ethnographie contemporaine et les généalogies nous montrent que c'est un peuple métissé, où les influences de groupes chaquéens comme les Toba et les Choroti (Manjui) ont été profondes, tant sur la Culture matérielle que sur les systèmes de croyance, même si la composante intégratrice de l'idéologie tupiguarani reste prépondérante, ainsi que l'ont décrite des auteurs comme Viveiros de Castro (1986), Silva Noelli (1999) ou, dans un contexte proche, Combès (1992 : 236) :

«Subsistant dans les mythes, le cannibalisme s'est aussi métamorphosé dans la téalité : "cannibalisme social" des Chiriguanos du Piémont andin qui, s'ils om abandonné l'anthropophagie rituelle au XVIIe siècle, om "absorbé" et "digéré" une culture entière, celle de leurs voisins et "esclaves" Chané. "

11 Notons enfin que la version, colportée par les Chiriguano du Paraguay à des fins politiques, selon laquelle les Ñandeva auraient été leurs vassaux comme les Chané, semble peu fondée en l'état actuel de mon enquête: des contacts ont existé ${ }^{4}$, mais il semble qu'ils aient relevé d'échanges inter-ethniques de communautés vivant dans des écosystèmes très différents (Chaco et Piémont andin); les Ñandeva avaient toujours la possibilité de se retirer au fin fond du Chaco en cas de conflit, ou de tentative d'assujétissement.

12 Traditionnellement, les Guarani-Ñandeva se divisaient en deux sous-groupes, aux différences dialectales légères, mais dont les pratiques de subsistance étaient fort différentes: Pykuijyiwa ware, groupe de l'intérieur nord-ouest du Chaco boréal (la zone sèche et aride autour de Nueva Asunción aujourd'hui), et Oruru ware, qui vivent plus près du Pilcomayo, principalement de la pêche (Gutiérrez 1995). Ce sont ces derniers qui sont les plus connus (Schmidt 1937), encore que Métraux (1946) ne les mentionne que fort peu...

13 Le groupe de Santa Teresita a émigré en janvier 1942 de Pykuljyzwa (Nueva Asunción) à Kamachu (Mariscal Estigarribia), dans le Chaco central, suite à une grande sécheresse qui détruisit toutes les plantations et amena une grande famine (González $1968: 260$ ); l'environnement y est un peu différent, mais surtout, la communauté a été cantonnée 
dans une mission exiguë, au voisinage obligé de deux autres communautés, Guarani occidentaux (Chiriguano) et Nivaclé5. L'absence de terres, mais surtout la possibilité de travail salarié sur la base militaire proche ou dans les estancias voisines ont profondément bouleversé la vie économique des Ñandeva dès l'arrivée dans la mission. Cette dernière a bien sûr déprécié toutes les valeurs indigènes pendant plus de quarante ans, bien qu'aujourd'hui un repli idéologique des missionnaires soit net, et qu'une plus grande tolérance -ou un désintérêt- se fasse jour depuis une dizaine d'années.

14 Même si, comme dans de nombreux villages amérindiens du Chaco, la fluidité résidentielle est grande, le Barrio San Lázaro de la Misión Santa Teresita (à $4 \mathrm{~km}$ de la ville de Mariscal Estigarribia) actualise de façon assez stricte la topologie sociale traditionnelle. Il s'agit d'un groupe local constitué d'un noyau de sœurs et d'un frère, ainsi que des filles et fils du tuwicha ("chef») Evaristo Benítez (Mbahave 1915?-1989) etde son épouse Cristina Benítez (Apyrakua 1918 ?), encore vivante. L'uxorilocalité, règle ancienne, est très majoritaire; si le tuwicha actuel (on l'appelle aujourd'hui le lider, hispano-anglicisme introduit récemment) Ramon Benítez a la charge de son père, les autres familles nucléaires sont constituées de ses sœurs, de leur maris, et de leurs enfants et petits-enfants. La parenté et l'organisation sociale n'ont pas encore été étudiées, mais les mariages entre proches parents sont strictement interdits; en particulier, une franche répugnance est exprimée lorsqu'on évoque les unions entre cousins (croisés et parallèles). On peut ainsi associer l'organisation sociale ñandeva à celle qui s'avère assez courante dans le Chaco et que décrivent Braunstein \& Miller (1999 : 10-11), sous réserve d'une étude complète à venir.

Ce que l'on produit, ce que l'on obtient

En ce qui concerne les techniques traditionnelles (agriculture, élevage, collecte, chasse), les éléments que nous présentons ici sont incomplets, car ils sont rarement observables à Santa Teresita: une enquête ethno-historique est nécessaire, autant qu'une ethnographie de la vie contemporaine chez les Guarani-Ñandeva de Nueva Asunción (Pykuijyiwa), cadre écologique d'origine et lieu central de cette culture.

Certaines questions sont pourtant essentielles, en particulier dans le domaine de l'agriculture. Les peuples du Chaco sont jusqu'à aujourd'hui considérés -pour les décideurs, les ONG, les anthropologues du développement, etc.- comme $d^{\prime}(e x-)$ chasseurs-cueilleurs, et pêcheurs pour ceux qui vivent près du Pilcomayo (Miller 1999). Pourtant, la somme de Métraux (1946), qui reste la compilation de référence des sources classiques, mentionne que presque tous connaissaient l'agriculture, une bonne partie l'élevage et que ceux qui ne les pratiquaient pas l'expliquaient par des raisons écologico-météorologiques.

17 Les Ñandeva, en "bons Guaranis » pourrait-on dire (Schaden 1998 : 55-66), valorisent avant tout dans leur discours l'agriculture -et l'élevage des moutons, chèvres et chevaux - lorsqu'ils se soumettent à l'exercice de mémoire consistant à présenter leur société d'avant le contact permanent (soit avant 1935).

18 À Santa Teresita, pourtant, l'agriculture moderne est aujourd'hui assez peu efficace, comme " délaissée » à regret, car peu productive et très aléatoire en raison du climat. Ce dernier est très irrégulier : on décrit bien souvent le Chaco comme un " enfer vert ${ }^{6}$ les inondations importantes succèdent aux sécheresses caniculaires, et ce de façon relativement imprévisible (dernière référence en date : Braunstein \& Miller 1999: 3), sans parler, pour les jardins, des dégâts dus aux nuisibles. En 2000, par exemple, les 
haricots ont bien poussé, mais toutes les cucurbitacées ont été mangées par les vers. Ces faits objectifs, répétés d'année en année - une autre fois, c'est le maïs qui sèche sur pied- sont énoncés par les Ñandeva avec une sorte de fatalisme triste. On doit pourtant tenter d'expliquer pourquoi cette agriculture a été supposée efficace, il y a soixante ans, et non plus aujourd'hui. Nous y reviendrons. Cette situation contemporaine est assez paradoxale puisque les Ñandeva se considèrent comme des agriculteurs/éleveurs, et en particulier des cultivateurs de maïs (avati): cette céréale (connue traditionnellement en plusieurs variétés) est aujourd'hui toujours cultivée, mais la production est insuffisante pour l'autoconsommation dans le cycle annuel. Elle n'en demeure pas moins importante, par exemple sur le plan rituel : le «carnaval»-parea, c'est-à-dire invitation- ne peut se concevoir sans bière kawî, préférentiellement de maïs. Parmi les autres cultigènes, on mentionnera jety, la patate douce de culture récente, andai et myndaka, deux cucurbitacées, kumada, le haricot, sandía (mot espagnol), la pastèque. Une chacra (mot espagnol d'Amérique : ici, un ensemble de champs contigus) collective est partagée en parcelles familiales, dans une zone sablonneuse ${ }^{7}$ située à environ $1 \mathrm{~km}$ du village proprement dit $^{8}$. Les travaux agricoles sont partagés entre hommes et femmes, sans qu'une spécialisation apparaisse clairement aujourd'hui dans cette répartition.

19 La cueillette est très peu pratiquée, car les Ñandeva de Santa Teresita ne vont plus guère en forêt, bien que la communauté soit propriétaire d'une parcelle à environ $17 \mathrm{~km}$ du village. Les caroubes (ywope wapehy ia) ne sont ramassées et consommées que par de rares personnes de plus de 50 ans. Les fruits de cactus cereus sp. (taringy) sont par contre assez régulièrement ramassés, séchés, puis servent à la confection d'une décoction mélangée à du piment sauvage (kyy). Seul le miel, ei, est encore récolté avec passion en forêt, pour la seule autoconsommation. Les ruches, dans le village, sont bien entretenues, et une bonne partie de la production est vendue; on peut même dire que c'est la seule activité économique traditionnelle qui se maintienne correctement, et en laquelle les Ñandeva voient des perspectives d'avenir.

Les animaux relèvent de la propriété individuelle : ils ont leur « maître », ija. L'absence prolongée de ce dernier dans le village ne remet pas en cause cette propriété. . L'analyse de ce terme polysémique ija dépasse le cadre de cet article; je ne puis donner ici que quelques éléments. C'est un concept qui va bien au-delà de la simple notion de " propriétaire » ou de « maître ", et qui est aussi connexe de ce que l'on traduit souvent improprement par " esprit ». Chaque classe d'êtres vivants possède, ou mieux, inclut son ija. Celui-ci a cependant une certaine autonomie, et peut en particulier agir en lieu et place de l'être animé en question, avec des propriétés ou qualités particulières. Par exemple, kururu ija, ija du crapaud, contrôle le tonnerre et la tourmente grâce au vecteur de son chant. Certaines classes d'êtres inanimés (jusqu'aux montagnes ou aux pierres, pourtant absentes du Chaco) ont elles aussi leur ija, souvent puissants. Un ija peut être acquis par un être animé d'une classe différente, là encore par le vecteur de son chant : par exemple, un paje (chamane) connaît et possède un ou plusieurs chants, ce qui lui permet de «faire agir " l'ija de telle ou telle entité. Les relations entre les êtres impliquent la relation, sous quelque forme que ce soit, de leur ija respectif. Posséder un animal domestique, c'est en fait plutôt connaître, et dans une certaine mesure contrôler, le ija de cette classe d'animaux, avec une application locale à certains individus. On devient ainsi, presque par abus de pouvoir, pourrait-on dire, soi-même chargé de cet ija, et donc en charge de ces animaux. 
21 Ainsi, la personne humaine "chargée et en charge » d'un animal ou d'un ensemble d'animaux domestiques est en fait dans une relation complexe avec celui-ci (ou ceuxci), qui s'inscrit dans le cadre plus général de la relation à l'animal.

On a vu que l'élevage des chèvres est le seul d'importance qui soit pratiqué dans la communauté. Le troupeau compte entre 10 et 30 têtes, suivant les moments: un nombre important de vieux mâles (4 ou 5) est associé à une majorité de femelles et de jeunes. Ce bétail est avant tout destiné à la vente, qui procure l'argent nécessaire à l'achat de nourriture, mais il est aussi dans une moindre mesure autoconsommé. Les animaux sont gardés dans un enclos de facture classique pour le chaco: des poteaux d'un mètre cinquante environ sont disposés côte-à-côte de façon serrée, formant une palissade robuste. Le matin, les chèvres sont lâchées dans le village et ses alentours et elles regagnent leur enclos le soir. Elles se nourrissent de la végétation épineuse, sans problèmes sanitaires particuliers. N'étant pas gardées, elles peuvent malheureusement aller jusqu'à la chacra, qui n'est pas complètement clôturée, et y produire des dégâts considérables; ce fait est présenté comme le principal frein au développement de leur élevage. Malgré les barrières d'épineux non comestibles placées sur leur chemin, elles arrivent à trouver des passages pour se rendre dans les plantations. Les animaux qui « connaissent » ces itinéraires doivent être vendus ou abattus.

Les cochons sont élevés par deux familles, et en petit nombre. Ils sont consommés lors de fêtes et partagés au sein de la parentèle. Le développement de leur élevage est limité par les possibilités d'achat d'aliments (maïs). Les poules sont plus répandues et font l'objet d'une pratique comparable, les œufs sont mangés assez régulièrement. Enfin, l'élevage du cheval et de l'âne a disparu, alors que l'image du cavalier guerrier, armé de sa lance mi, est toujours présente dans les gestes des combats héroïques contre les Boliviens ou les Ayoreo. Les chiens sont en revanche assez nombreux, sans doute moins qu'à l'époque des observations de González en 1942-43 (1968 : 279).

Les perroquets apprivoisés sont également beaucoup moins présents; seule une famille continue d'en entretenir. Ils étaient, jusqu'au milieu des années 1980, capturés et gardés pour la vente aux Néo-américains. L'interdiction de ce commerce (CITES, appendices 1 ou $\mathrm{II}^{10}$ ) n'en a pas complètement tari le trafic, mais les Nandeva de Santa Teresita ont délaissé cette activité. Le nddu (Rhea Americana) est le seul animal sauvage "domestiqué »: capturé en bas âge lors des chasses, il est élevé dans le village, et consommé pour sa chair.

Reste maintenant à expliquer comment deux activités - l'agriculture et l'élevageinscrites dans la tradition et symboliquement valorisées, sont soit en déclin, soit en crise. On peut imputer un faisceau de causes.

Tout d'abord, la raison évoquée pour les échecs agricoles par les Ñandeva eux-mêmes n'échappera pas au musicologue: c'est l'absence, aujourd'hui, de grands chamanes capables d'exécuter les chants pour la pluie (types de chants que l'on trouvait dans tout le Chaco), ou de chants propitiatoires spécifiques. Le savoir chamanique s'est partiellement perdu à cause des missionnaires, mais aussi, nous dit-on, parce que les jeunes n'étaient, il y a une trentaine d'années, pas assez conscients de l'immense valeur des savoirs des Anciens. Déficit cosmologique, donc. C'est l'absence de maîtrise des ija en relation à la pluie qui ne permet plus aujourd'hui aux Ñandeva de " gérer » la venue des précipitations. 

ayant suivi la missionnisation, un transfert des activités classiques vers le salariat précaire du secteur agricole ${ }^{11}$-en espagnol local, la changa-, principal moyen d'obtenir de l'argent dans le Chaco. Abondamment décrite (Fritz 1994; Renshaw 1996; entre autres), Cette changa ne diffère en rien de celle pratiquée par les autres groupes. Colonies mennonites (en particulier la colonia 5) ou estancias procurent des travaux salariés en général instables et mal payés... Remarquons simplement que la connaissance des milieux très arides du Chaco Boréal, que les Ñandeva partagent avec leurs ex-ennemis Ayoreo, en font souvent des salariés prisés pour travailler au nordouest de Mariscal Estigarribia. Sa proximité joue évidemment un rôle majeur; cette petite ville, qui s'est développée autour d'une base militaire, fournit un travail à nombre de journaliers indigènes en dehors de la classique changa. Mais la base rassemble de moins en moins de soldats, et l'emploi stable ${ }^{12} \mathrm{y}$ a pratiquement disparu pour les personnels non militaires. Historiquement, l'emploi de salariés indigènes a pourtant été fondamental dans l'histoire de l'intégration des Ñandeva à l'économie de marché. En particulier, la briquetterie militaire a employé beaucoup de Ñandeva, considérés comme "travailleurs et disciplinés ». Seuls deux salariés du Barrio San Lázaro sont aujourd'hui ouvriers permanents dans cette activité. D'autre part, une sorte de clientélisme s'est établi entre des gradés et certains Ñandevas : les militaires emploient les Amérindiens pour de menus travaux, les Amérindiennes pour les tâches domestiques. Empreintes de paternalisme, ces relations permettent néanmoins à quelques familles de gagner un peu d'argent ou d'obtenir quelques avantages (rations, etc.).

Bien sûr, l'exiguïté de la mission est aussi une donnée fondamentale qui explique en particulier la régression de l'élevage des moutons et des chèvres, mais nous pensons pouvoir développer l'hypothèse que l'agriculture, inscrite profondément dans le patrimoine culturel commun des Guarani, et donc idéologiquement structurante de la culture ñandeva, n'a en fait jamais été suffisante, même opportunément jointe à l'élevage, dans les conditions particulières du Chaco ${ }^{13}$.

Il nous faut intégrer les dimensions ethno-historiques, et en particulier le fait suivant: bien avant le contact permanent avec les Néo-américains, dès les années 1880, les hommes ñandeva de Pykuijyiwa allaient à mbaporenda -le « lieu du travail », c'est-à-dire en Argentine- louer leurs services dans les ingenios (raffineries) de canne à sucre (pour le cadre général, voir Braunstein \& Miller 1999 : 15-16). Les hommes partaient cinq ou six mois, laissant leur famille dans les régions, alors inaccessibles, qui entourent Pykuijyiwa. D'une part c'est là, sur ces lieux de travail, que s'effectuèrent sans doute une bonne partie des échanges inter-ethniques avec les Choroti (Manjui) et les Toba, et d'autre part, on peut penser que les hommes partaient ainsi travai11er parce que, déjà, l'agriculture et l'élevage ne suffisaient pas toujours à assurer la subsistance. Antérieurement encore, il est probable que les Ñandeva de Pykuijyiwa aient participé à des échanges occasionnels avec les Chiriguano, troquant leur force de travail (pour les récoltes) contre du maïs.

Dans les deux cas cités, les Ñandeva maîtrisaient assez largement ces relations car ils en avaient l'initiative exclusive; jusqu'à la fin du siècle dernier, leurs alliances guerrières avec les Toba (mentionnées par Thouar 1891) devaient décourager les plus téméraires de leurs poursuivants, sans compter l'extrême aridité de la région. Ainsi, agriculteurs structurellement déficitaires, les Ñandeva auraient dû pratiquer à un haut degré -mais

Techniques \& Culture, 38 | 2002 
compatible avec le "cannibalisme social» tupi-guarani (Combès 1992) l'intégration d'éléments allogènes de divers ordres : matériels, immatériels (la musique en est un exemple) ou matrimoniaux.

31 Dans la société ancienne, il ne semble pas que la chasse ait été une activité essentielle, même si elle était très valorisée. Traditionnellement, on pratiquait la chasse et la consommation du pécari (tayasu), du daguet (wasu), du tapir (mborevi), du nandou (ñadu) et du guanaco (wasu puku). Certaines espèces, comme le tatou boule (tatu) et le fourmilier (apyrakwa) étaient collectées ou chassées, mais réservées à la consommation des anciens (hommes et femmes). Le jaguar (jawa) et le puma (jawa pyta), quant à eux, n'étaient pas consommés - normalement-, car ils rendent furieux, dit-on, la personne qui les mange. Aujourd'hui, la chasse, dont l'importance économique est limitée, se résume à deux formes.

32 1) Autour de la chacra, il y a une zone d'environ 1 à $2 \mathrm{~km}$ où vivent les animaux qui se nourrissent des plantes cultivées. On les chasse à la carabine 22 long rifle (plus rarement au fusil calibre 12) dans la chacra ou près d'elle, dans les haies, ou un peu plus loin dans la forêt, là où il $\mathrm{y}$ a un nombre important de chemins utilisés pour aller chercher le bois de chauffe. Cette chasse de proximité est de loin la plus pratiquée, parfois seul, ou à deux ou trois, et ce toujours sans chiens. Les espèces prélevées sont les oiseaux jeruti (Leptofifa sp.), arâkua (Ortallis canicollis pantanafensis), le lièvre tapiti (Syfvifagus brasifiensis), le daguet wasu (Mazama americana) ${ }^{14}$, et ajatutu (tuka tuka en guarani jopara, sorte de taupe ? ${ }^{15}$.

33 2) Quand des moyens de transport sont disponibles (moto, camionnette), des chasses plus lointaines (20 à $30 \mathrm{~km}$ de Mariscal Estigarribia) sont effectuées, permettant la prédation de wasu ou de tayasu.

34 Les grandes chasses traditionnelles à cheval du wasu puku (guanaco, Lama guanicoe) et du ñãdu (Rhea americana) ne sont plus pratiquées ${ }^{16}$. La vente d'animaux considérés par les Paraguayens -et en particulier par les militaires- comme de compagnie (perroquets, singes) a pu constituer un apport d'argent.

Enfin, les remarquables dessins d'animaux et de scènes de chasse, peints sur les murs des premiers temps de la missionnisation (González 1968; 280281-330), ont malheureusement disparu de la culture ñandeva avec les artistes de l'époque.

Terminons en disant que pour les Pykuijyiwa ware, la pêche et la consommation du poisson semblent pratiquement inconnues, tant dans la société traditionnelle que dans la société contemporaine, du fait de l'absence de fleuve ou d'étang de taille suffisante.

C'est donc grâce aux achats de denrées alimentaires, à la coopérative militaire ou dans les commerces de Mariscal Estigarribia, que les Ñandeva assurent aujourd'hui une bonne part de leur subsistance. L'argent nécessaire provient très largement de l'emploi précaire. À titre d'exemple, une unité domestique peut disposer de revenus variant de $100000 \mathrm{C} \$$ (environ 30 euros) mensuels pour les plus pauvres à $400000 \mathrm{C} \$$ (environ 120 euros) pour une famille où un salaire régulier d'ouvrier-briquetier est touché chaque mois. On verra plus bas qu'en fait, ces données ne permettent pas d'approcher la réalité de ce dont disposent les individus : un système traditionnel et efficace de redistribution obligée de denrées alimentaires fonctionne et nivelle ce qui pourrait apparaître de prime abord comme un cadre inégalitaire de revenus. Selon les périodes, les achats sont d'importance inégale, selon la production de la chacra. Disons qu'entre juillet et février, leur importance est fondamentale : on achète principalement du riz, des pâtes, de la 
farine de blé, du sucre, du maté et de l'huile, indispensables à la diète actuelle. Parfois, lorsqu'on le peut, des haricots et de la viande de bœuf (bas morceaux vendus sous le nom de puchero) complètent le panier.

Le commerce

Le numéraire est donc indispensable à la satisfaction des besoins alimentaires. L'achat de la nourriture se fait dans les magasins privés ou dans la coopérative militaire. On vend à crédit dans les deux cas, avec des systèmes comparables; la coopérative a, par exemple, mis en place un système de livret, permettant de gérer le crédit sur le compte de l'ouvrier, et de déduire directement la dette sur le salaire. Il va de soi que lorsque le client atteint un certain seuil d'insolvabilité du point de vue du commerçant, le crédit s'arrête.

Il est intéressant, à ce stade, de dire quelques mots de la perception qu'ont ces petits commerçants de la consommation indigène. Nous sommes dans le cadre du paradigme général de la vision des Amérindiens par les Néo-américains: celui d'une forte dépréciation des Amérindiens, à la fois méconnus, méprisés et parfois craints par les Néo-américains. Ils sont classés dans la catégorie unique indígenas, alors que l'on a affaire, dans le Chaco, à des cultures et à des groupes linguistiques différents, malgré une certaine homogénéité dans les strates les plus superficiellement observables de ces sociétés (culture matérielle traditionnelle, par exemple).

Ce qui revient le plus souvent dans le discours fréquemment indirect et sans doute assez retenu ${ }^{17}$ des commerçants sont les qualifications négatives d'imprévoyance ${ }^{18}$ et de goût pour la soûlerie : il est intéressant de constater que, d'une part, c'est justement cette imprévoyance supposée qui permet les pratiques usuraires de la dette à forte valeur ajoutée, et que, d'autre part, la vente d'alcool aux Amérindiens représente une part non négligeable du chiffre d'affaires ${ }^{19}$.

41 Nous allons essayer de qualifier pour partie la logique culturelle qui amène à ces comportements considérés comme imprévoyants dans les stratégies de consommation; pour cela, revenons au système de la dette : il ne fonctionne pas avec un taux de crédit, impliquant des remboursements mensuels de la dette et de ses intérêts, mais par une augmentation des prix de détail et par l'ouverture d'une " ardoise ». Ces augmentations sont considérables : un kilo de riz, par exemple, est vendu $2500 \mathrm{C} \$$, alors qu'acheté comptant au commerçant paraguayen de Mariscal Estigarribia, il revient à $1300 \mathrm{C} \$$, lorsqu'on l'achète par 30 kilos. Comment expliquer alors que les Ñandeva, même s'ils ne «calculent» pas $^{20}$, n'ignorent pas cet état de fait et continuent de se faire ainsi exploiter (selon les critères objectifs de l'économie comptable)? Observons les stratégies de consommation.

La consommation alimentaire : que partage-t-on?

Le cadre général est celui que décrit Renshaw (1996: 145-] 69): forte idéologie égalitaire, importance accordée au libre arbitre des individus, existence d'une pratique de la propriété individuelle de certains biens, pratique de l'«impossibilité de refuser » qui dépasse la réciprocité à court terme (voir aussi pour les Enxet -Lengua-, Kidd 1999 : 41-48). Les échanges dont nous allons parler ont lieu strictement au sein du groupe local, éventuellement étendu aux Ñandeva "de passage ", liés par la parenté aux groupes du Barrio San Lázaro.

La nourriture, c'est-à-dire les aliments transformés par la cuisine, appartient à celle qui l'a élaborée et à sa maison: par exemple, l'expression "hêêête ry moiva» (c'est bon, ta nourriture) est employée pour exprimer la qualité et la provenance de la préparation. 
Cette propriété fait qu'il est rarissime de constater un don de nourriture à emporter : celui qui a faim vient manger dans l'espace privé et dans le cadre des repas collectifs, mais ne peut emporter la nourriture dans son propre espace familial. Prenons l'exemple des principaux ingrédients qui composent un menu.

Deux ou trois repas sont pris (matin, midi, soir), selon les disponibilités en nourriture. Sur trois jours d'un mois avec autoproduction agricole (soit de janvier/février à avril/ mai), les aliments suivants peuvent être consommés :

- jour 1 : pastèque (chacra), haricots (chacra), riz (commerce), maté (commerce); fruits de cactus frais ou séchés avec du piment (collecte);

- jour 2 : œufs (du village), courge (chacra), puchero (bas morceaux de bœuf), oignon (chacra), maïs (chacra), poivron (chacra), maté chaud ou froid (terere);

- jour 3 : pastèque, maïs, pâtes (commerce), salade (chacra), maté;

En revanche, sur trois jours d'un mois sans autoproduction agricole (soit de mai/juin à décembre/janvier) :

- jour 1 : pâtes, locro (maïs doux séché, commerce), maté;

- jour 2 : galettes de farine de blé frites, miel (ruches), pain (commerce), maté;

- jour 3 : riz, puchero, maté.

On constate à la vue de ces menus que la saison sèche, sans production agricole, impose une dépendance très grande par rapport aux aliments achetés: c'est une période difficile, où il n'est pas rare que la faim menace.

Par beau temps, la cuisine est faite dehors, sur un feu de bois dont l'emplacement n'est pas fixe. Par mauvais temps, un abri-cuisine, bâti à côté des maisons, est utilisé pour protéger le foyer des intempéries. Le four (en brique, d'origine néo-américaine) est établi à quelques dizaines de mètres de là : il sert à la préparation de viande rôtie (rarement disponible) ou de plats paraguayens. proche est employé, à l'exclusion d'autres combustibles (tous les résidus ou déchets de produits transformés sont strictement prohibés ${ }^{21}$ ). C'est toujours à la mère et à ses filles que revient la charge de l'approvisionnement en eau et la préparation de la nourriture, alors que les hommes vont en général chercher le bois de chauffe. Les aliments sont principalement bouillis, la viande étant parfois frite (quand il y a de l'huile du commerce) avant l'ajout de liquide. Les aliments cuits sont en général servis dans un bouillon (y compris les pâtes ou le riz). D'après ces descriptions, on peut dire que les menus des Ñandeva de Santa Teresita ne sont pas vraiment différents de ceux des Néoaméricains pauvres du Chaco.

D'un point de vue sociologique, ce sont surtout les proches qui s'invitent; manger ensemble, c'est affirmer son adhésion à un réseau de proximité. Par exemple, suite à une dispute, le fait de venir manger dans la maison du camp adverse signifie le désir de mettre fin à la brouille. Cela se fait toujours sans invitation, et le repas, vite pris, est accompagné de commentaires généraux assez formels, sans aucune allusion au conflit que l'on souhaite apaiser.

51 Différente est la circulation des aliments : ce sont des biens que l'on peut donner, et surtout demander sans que l'on s'implique dans un réseau social ou un réseau d'alliance politique particulier. Bien sûr, un tropisme d'affinité (au sens commun du terme) existe, selon les moments : on demandera plus à telle ou telle personne, mais cela ne constituera pas la base d'un réseau de relations préférentielles. Le cadre idéologique est 
clairement énoncé : «Celui qui a ne peut pas refuser de donner; il peut choisir, par contre, de donner plus ou moins ». Avec les conséquences que l'on peut attendre -soit une demande importante est satisfaite, et donc pour un laps de temps assez long, soit l'on s'expose à des demandes répétées. Toutes les interactions sont possibles dans ce cadre, mais les refus ne peuvent être directs. C'est pourquoi l'accumulation d'aliments est rendue difficile; par exemple, une famille qui aurait acheté le riz par sacs de $30 \mathrm{~kg}$ s'expose aux demandes de dons de ceux qui en manquent -et il y en a toujours, dans cette économie de la misère. Demandes qu'elle ne pourra, avec des trésors d'ingéniosité, que différer. Le stockage ne se pratique pas, et l'on achète au fur et à mesure des besoins, même à un prix déraisonnable. Qu'en est-il dans le domaine des productions agricoles et de l'argent?

52 Les parcelles cultivées sont de taille comparable quelle que soit la famille, les cultigènes comme le climat, identiques, et les périodes d'abondance ou de manque sont synchrones. Tout au plus constate-t-on de légers décalages d'une ou deux semaines entre les périodes de plantations et de récoltes (pastèques, haricots ou maïs). La quantité de main-d'œuvre selon les llilités domestiques joue peu car elle est plus ou moins équivalente.

L'argent est toujours rare. Le fait d'en emprunter ou d'en demander le don n'est donc que marginal. La « mentalité de l'aubaine » (windfall mentality) Hugh-Jones cité par Kidd 1999 : 49) existe fortement chez les Ñandeva : les biens manufacturés, même les plus «inutiles» ou "saugrenus» selon notre vision de l'efficacité économique et des logiques de réduction de la misère, exercent un attrait irrésistible lorsque l'on a la poche suffisamment remplie pour se les procurer. Cependant, contrairement à la nourriture, mais comme les biens personnels (au sens de Renshaw 1996 : 157-158), on peut refuser le don ou le prêt d'argent.

Il est enfin remarquable de constater que dans cette société villageoise, les conflits ${ }^{22}$ ne sont pratiquement jamais liés à des questions d'accès aux aliments: sur le seul plan économique, les antagonismes existent, mais ils se situent au niveau de la production des biens (par exemple, malveillance envers une portée de jeunes cochons), ou de la répartition considérée comme inéquitable (ainsi, des vêtements offerts par une ONG).

Somme toute, dans une communauté comme celle du Barrio San Lázaro de Santa Teresita, les formes traditionnelles de production des biens sont en très forte régression, voire disparaissent complètement. Contrairement aux Ngaatjatjarra d'Australie dont nous parle Dousset (1999), mais aussi à leurs voisins Ayoréo, Nivaclé ou Choroti, les Guarani-Ñandeva ne réactualisent plus leurs savoirs techniques dans la réalisation de leur culture matérielle. Pourtant, la notion d'« opportunisme » reste centrale dans leur vie économique. Travaux précaires, clientélisme discret auprès des militaires ou des politiques remplacent ici la « collecte en milieu urbain » décrite chez les Wichi ou les Weenhayek par Picon (1998) et Alvarsson (1999). Cet opportunisme, trop facilement regardé et brocardé comme un des avilissements les plus notables consécutifs aux processus d'acculturation, commence à être analysé, par ces auteurs et quelques autres, dans une logique historique où la prédation a changé d'objet, mais non de méthodes. En ce qui concerne les Guarani-Ñandeva, on remarquera que leur culture a dû faire preuve d'une constante adaptabilité, bien avant le contact permanent avec les Néo-américains: adoption de l'élevage au XVIIe ou XVIIIe siècle, recherche du travail salarié loin des villages dès la fin du XIXe siècle. Pour reprendre les termes de Picon (1998: 390), c'est bien vers la flexibilité, et non plus vers l'anomie ou la 
déstructuration que l'ethnologie commence à penser les réponses contemporaines que nous présentent les ex-chasseurs-cueilleurs. On voit avec les Guarani-Ñandeva que ce type de réflexion trouve un écho harmonique chez ces " agriculteurs malheureux », si tant est que l'on puisse ainsi évoquer le destin de cultivateurs de maïs dans la Chaco...

Bien que sans les lier explicitement à une notion d'« amour » de l'autre comme le font les Enxet (Kidd 1999 : 44-47, 49), les Guarani-Ñandeva de Santa Teresita actualisent de façon tout à fait manifeste les valeurs morales traditionnelles de partage et d'égalité quant aux besoins vitaux de l'alimentation. Leur économie, aujourd'hui très fortement intégrée au système monétaire standard, respecte, au niveau de la consommation, l'impérieuse nécessité de ne laisser personne sur le bas-côté. En cela, mais nous ne : sommes bien sûr ni les premiers ni les derniers à le dire pour le Chaco, les Ñandeva se distinguent radicalement des Néo-américains, avec qui ils partagent pourtant de grandes difficultés d'accès au travail décent

\section{BIBLIOGRAPHIE}

Alvarsson, Jan-Ake

1988. The Mataco of the Gran Chaco: An Ethnographic Account of Change and Continuity in Mataco SocioEconomic Organization. Uppsala Studies in Cultural Amhropology 11. Uppsala and Stockholm : Almqvist and Wiksell International. 1999. "Foraging in Town : Survival Strategies among the Weenhayek of Bolivia and Argentina ", pp. 22-35, in E.S. Miller (ed.), Peoples of the Gran Chaco. London : Bergin and Garvey.

Braunstein, José \& Elmer S. Miller

1999. «Ethnohistorical introduction », pp. 1-22, in E.S. Miller (ed.), Peoples of the Gran Chaco. London : Bergin and Garvey.

Combès, Isabelle

1992. La Tragédie cannibale chez les anciens Tupi-Guarani. Paris : Presses Universitaires de France (« Ethnologies»).

Del Campana, Domenico

1902. « Cenni su i Tapîi ed i Tapihete », Archivio per l'antropologia e la etnologia (Firenze) 32/2 : 283-289.

Dietrich, Wolf

1992. El idioma chiriguano. Madrid : Ediciones cultura hispanica, Instituto de Cooperacion Iberoamericana.

Dousset, Laurent 1999. «Que reste-t-il du social quand on ne chasse plus ? Les Ngaatjatjarra du désert de l'ouest australien », Techniques \& culture $33: 1-27$.

Fritz, Miguel

1994. Los Nivaclé; rasgos de una cultura paraguaya. Asuncion/Quito : co-édition Instituto Leon Cadoganl Ediciones Abya-Yala. 
González, Gustavo

1968. Entre los Guarani-chané (o Ñañagua) dei Noroeste Chaquefio », Suplemento Antropologico de la Revista dei Ateneo Paraguayo (Asunción) 3/1-2 : 259-338.

Gutierrez, Ramiro

1995. "Ernografia chaquefia, el caso de los Tapiétés dei Pilcomayo », Revista dei Museo Nacional de Etnografia y Folklore 6 : 74-106.

Kidd, Stephen W.

1999. Morality of Enxet People of the Paraguayan Chaco and their resistance to assimilation ", in E.S. Miller (ed.), Peoples of the Gran Chaco. London : Bergin and Garvey.

Métraux, Alfred

1946. « Ethnography of the Chaco », pp. 197-170, in L. Steward (ed.), Handbook of South American Indians, vol. 1. Washington : Smithsonian Institution, Bureau of American Ethnology.

1982. Les Indiens de l'Amérique du Sud. Paris : A. M. Métailié (« Traversées ») (1ère édition 1946).

Nordenskiöld, Erland

1910. « Sind die Tapiété ein guaranisiener Chacostamm? », Globus (Braunschweig) Bd. XCVIII :

181-196.

Picon, François-René

1998. « De la collecte en milieu urbain chez les Mataco (Chaco argentin) », Techniques \& culture

$31-32: 379-395$

Renshaw, John

1996. Los Indigenas dei Chaco paraguayo, Economia y Sociedad. Asuncion : Intercontinental Edirora.

Schaden, Egon

1998. Aspectos fündamentales de la cultura guarani. Asunción : Biblioteca Paraguaya de Antropologia, vol. 28, Universidad Catolica (lère édition 1954).

Schmidt, Max

1937. «Los Tapiétés », Revista de la Sociedad Cientifica dei Paraguay (Asunción) IV/2 :36-67

Silva Noelli, Francisco

1999. "Cun Nimuendaju e Alfred Métraux : a invençao da busca da "terra sem mal" ", Suplemento Antropológico XXXIV, 2 : 123-167.

Thouar, Auguste

1891. Exploration dans l'Amérique du Sud. Paris : Hachette.

Viveiros de Castro, Eduardo

1986. Os deuses canibais. Rio de Janeiro : Jorge Zahar.

Von Bremen, Volker

1991. Zwischen Anpassung und Aneigung Zur Problematik von Wildbeuter-Gesellschaften im modernen Weltsystem am Beispiel der Ayoreode. München : Münchener Amerikanistik Beirrage Nr 26. anaconVerlag

\section{NOTES}

1. Connus dans la littérature sous le nom de Tapiétés; l'autodénomination à usage externe Guarani-

Ñandeva est récente (années 1980) et due aux conflits d'identité avec les Guarani 
occident aux (Chiriguano), essentiellement dans le Chaco central. Elle est propre au Paraguay. L'autodénomination à usage interne est plutôt Kwareta ou Ñandereta 2. Bien rédigée en 1981, quelques années avant la fin de la dictature, mais peu ou mal appliquée dans les faits, cette loi garantit de nombreux droits aux Amérindiens, et en particulier l'indivisibilité el l'incessibilité des terres communautaires.

3. La plupart des auteurs récents entérinent cette c1assitication : Renshaw (1996) ou, par exemple Braunstein et Miller : « The Gran Chaco peoples have been huntergatherers, in contrast to their agriculturalist neighbors on the western border " (1999: 5). Il serait certainement utile de revisiter cette classification, qui reste à la base des constructions visant à expliquer le comportement actuel de ces cultures. Métraux (1946) avait pourtant une position plus nuancée et, plus récemment, Picon (1998 : 385-386, 389), en particulier, a réinterrogé la c1assitication et notamment la notion de collecte dans le Chaco argentin.

4. Il est probable que des Chiriguano mentionnés comme «tllyam dans le Chaco » lors des nombreuses guerres avec les Espagnols puis les Boliviens (jusqu'en 1892), se sont joints aux Ñandeva, l'intégration des groupes étant facilitée par l'évidente proximité linguistique. De plus, des Ñandeva ont dû travailler occasionnellement chez les agriculteurs du Piémont.

5. Cette cohabitation est une source de conflits plus ou moins activés selon les moments. L'opposition Guarani-Ñandeva/Guarani Occidentaux (Chiriguanos) est fortement marquée, malgré un certain nombre de récents mariages interethniques.

6. Métraux (1982 : 77-78) en nuançait les termes, dans un article qui reste à ce jour la meilleure introduction aux sociétés traditionnelles de cette région. En particulier, le paragraphe sur les rêves demeure d'une forte actualité.

7. Ce sont les seules, dans le Chaco central et boréal, qui se prêtent un tant soit peu à l'agriculture : les autres sols, argileux, sont beaucoup trop lourds pour permettre leur exploitation.

8. La municipalité de Mariscal Estigarribia aide aux labours grâce au prêt d'un tracteur, une fois par an.

9. Nous avons deux exemples (chèvres et cochons) de cette propriété, maintenus malgré des absences de plusieurs mois ou années : le « maître » de chèvres vit en effet en Argentine, sans que sa propriété ne soit remise en cause. La gestion du troupeau revient à son frère, désigné par lui pour le remplacer.

10. La Convention on International Trade in Endangered Species, datant de 1988 11. Il n'existe dans le Chaco presque aucun petit paysan possédant et exploitant des terres à l'échelon familial. Le système de propriété foncière et de colonisation mis en place après la guerre de la Triple Alliance (années 1880), puis après la guerre du Chaco (années 1940), a toujours privilégié les grands ensembles latifundiaires lors de la vente des terres de l'État.

12. Dans les années 1940-50, des Ñandeva étaient employés dès l'âge de 12 ans comme ouvriers permanents.

13. Des changements climatiques brutaux (on ne dispose pas d'études scientifiques à ce sujet) peuvent être également hypothétiquement évoqués pour rendre compte des faibles rendements de l'agriculture et de l'élevage. Nous savons en effet que, par le passé, des sécheresses ont profondément bouleversé l'existence des communautés guarani-Ñandeva (celle de 1942 en particulier),

14. Ce dernier est le plus difficile à tuer car c'est un animal craintif et rapide. On le trouve également plus loin dans la forêt, mais il peut faire l'objet d'une chasse 
spécifique en effet, il aime s'approcher des micro-salines pour lécher le sel sur la terre. En s'approchant très doucement, on peut espérer le rencontrer et le surprendre.

15. C'est le seul animal encore parfois chassé avec l'arc Wvrapa et les flèches on l'attend devant la sortie de son terrier.

16. De même, àguja wasu (viscache, Lagostomus maximus), qui donne traditionnellement lieu à une prédation dans les zones de culture, n'est pas présent dans la région de Mariscal Estigarribia, alors qu'il reste une prise importante pour la communauté de Pvkuijy'iwa.

17. En général, lorsqu'il s'agit de parler affaires et en particulier envers l'« Européen riche venu défendre et aider les indigènes ".

18. Remarquons que les réflexions les plus communément entendues : «Ils dépensent tout leur argent quand ils en ont ", « ils ne payent pas leurs dettes », «Ils achètent des choses inutiles et manquent ensuite de nourriture », sont partagées par les commerçants (qui en profitent) et par les missionnaires, ONG, services sociaux (qui le déplorent, renforçant ainsi la position symbolique d'Autre radidal des Amérindiens). On verra plus loin que cette supposée imprévoyance participe en fait d'une répartition égalitaire des denrées.

19. Cet état de fait mériterait une analyse anthropologique fine et approfondie, dépassant les commentaires habituels, banals et moralisateurs. Par exemple, la relation musique/alcool souvent mise en valeur dans les cultures amérindiennes, doit être spécifiée pour les cultures du Chaco.

20. Au sens naïvement ethnocentrique du terme : il n'existe en effet pas d'études sérieuses sur les mathématiques indigènes dans le Chaco. Pourtant, les procès de catégorisation qualitative (taxonomie) et quantitative (calcul) devraient être étudiés, en particulier pour des applications dans le domaine de l'aide au développement durable que l'on dit souhaité par de nombreuses ONG.

21. Même les enveloppes de sachets de thé...

22. La question de la sorcellerie a une importance considérable dans la vie quotidienne : c'est à elle, par l'Intermédiaire des esprits malveillants envoyés par un chamane spécialisé (et toujours inconnu), que sont attribuées les infortunes les plus diverses de la vie Individuelle ou collective.

\section{RÉSUMÉS}

Depuis 50 ans, des familles d'indigènes guarani-ñandeva (appelés aussi Tapietes) sont installées dans la communauté de Santa Teresita, dans le Chaco central paraguayen. Si l'économie et l'alimentation traditionnelles ont été profondément modifiées, la consommation obéit toujours aux principes égalitaires traditionnels. Les modalités de partage permettent à tous d'accéder à la nourriture, et empêchent l'accumulation de denrées alimentaires dans les mains de certaines personnes.

Culture and consumption in Guarani-Nandeva community in the Chaco.

Figty years ago, some families of Guarani-Ñandeva (or Tapietes) indians settled in the community of Santa Terisita in the Central Parayuayan Chaco. If their traditional economy and food habits 
have been deeply modified, nevertheless, the modalities of consumption are still ruled by egalitarian principles allowing access to food for all while preventing its acculturation in the hands of certain individuals.

Cultura y consumo en una comunidad Guarani-ñandeva del Chaco.

Hace 50 años, unas familias de indígenas Guarani-Ñandeva (también llamados Tapietes) se instalaron en la comunidad de Santa Teresita en el Chaco central paraguayo. Tanto la economía como la alimentación tradicionales han sido profundamente modificadas. Sin embargo, el consumo obedece todavía a los principios egalitarios que permiten a cada persona el acceso a la comida y, al mismo tiempo, impiden que se acumulen alimentos en las manos de unos cuantos individuos.

INDEX

Mots-clés : alimentation, Chaco, consommation, Guarani, indigènes

Keywords : consumption, food, indigenous

\section{AUTEUR}

JEAN-PIERRE ESTIVAL

UMR 8574 CNRS/Université Paris X/MNHN, Musée de l'Homme, 17 place du Trocadéro 75116 Paris. 